\title{
AM 1934-563: a giant spiral polar-ring galaxy in a triplet ${ }^{\star}$
}

\author{
V. Reshetnikov ${ }^{1,2,4}$, F. Bournaud ${ }^{2}$, F. Combes ${ }^{2}$, M. Faúndez-Abans ${ }^{3}$, and M. de Oliveira-Abans ${ }^{3}$ \\ 1 Astronomical Institute of St. Petersburg State University, 198504 St. Petersburg, Russia \\ e-mail: resh@astro.spbu.ru \\ 2 Observatoire de Paris, LERMA, 61 Av. de l'Observatoire, 75014 Paris, France \\ 3 MCT/Laboratório Nacional de Astrofísica, Caixa Postal 21, CEP:37.504-364, Itajubá, MG, Brasil \\ ${ }^{4}$ Isaac Newton Institute of Chile, St. Petersburg Branch
}

Received 26 July 2004 / Accepted 15 September 2005

\section{ABSTRACT}

We have observed the emission-line kinematics and photometry of a southern triplet of galaxies. The triplet contains a giant spiral galaxy AM 1934-563 whose optical structure resembles a polar-ring galaxy: a distorted spiral disk, seen almost edge-on, and a faint large-scale (45 kpc in diameter) warped structure, inclined by $60^{\circ}-70^{\circ}$ with respect to the disk major axis. The triplet shows a relatively small velocity dispersion $\left(69 \mathrm{~km} \mathrm{~s}^{-1}\right)$ and a large crossing time (0.17 in units of the Hubble time). The disk of AM 1934-563 demonstrates optical colors typical of early-type spirals, a strong radial color gradient, and almost exponential surface brightness distribution with an exponential scalelength value of $3.1 \mathrm{kpc}$ ( $R$ passband). The galaxy shows a maximum rotation velocity of about $200 \mathrm{~km} \mathrm{~s}^{-1}$ and it lies close to the Tully-Fisher relation for spiral galaxies. The suspected polar ring is faint $(\mu(B) \geq 24)$ and strongly warped. Its total luminosity comprises $10-15 \%$ of the total luminosity of AM 1934-563. We model this system using numerical simulations, and study its possible formation mechanisms. We find that the most robust model that reproduces the observed characteristics of the ring and the host galaxy is the tidal transfer of mass from a massive gas-rich donor galaxy to the polar ring. The physical properties of the triplet of galaxies are in agreement with this scenario.

Key words. galaxies: individual: AM 1934-563 - galaxies: interaction - galaxies: formation - galaxies: structure

\section{Introduction}

Polar-ring galaxies (PRG) are peculiar objects in which a polar or very inclined ring surround the host galaxy. They are very informative about galaxy formation. At least two main scenarios are invoked to form these systems: either the major merger scenario, with a head-on merging of two galaxies with their disks oriented perpendicularly (e.g., Bekki 1998), or the accretion scenario, where a gas-rich donor loses matter to form the ring (e.g., Schweizer et al. 1983; Reshetnikov \& Sotnikova 1997). The accretion scenario itself could either correspond to a small companion disrupted during a minor merger, or a major encounter with tidal mass transfer from a massive donor to the host (Bournaud \& Combes 2003, hereafter BC03). In previous simulations, $\mathrm{BC} 03$ have shown that the accretion scenario offers more chances of forming the observed systems, and that a discriminating characteristic can be the existence, in the merger scenario, of a diffuse stellar background around the polar-ring system. In the accretion scenario, the matter is not as dispersed spatially. It is important to test the possible scenarios on several

^ Based on observations made at the Observatório do Pico dos Dias (OPD), operated by the MCT/Laboratório Nacional de Astrofísica, Brazil. well-observed polar-ring systems and the present system offers a good example.

The southern peculiar galaxy AM 1934-563 was classified by Whitmore et al. (1990) (PRC, polar rings catalogue) as a good candidate polar-ring galaxy. (The object name is PRC B-18 according to the PRC.) The faint extended feature crosses the main galaxy (disturbed edge-on galaxy with inclined dust lane) at an angle of about $60^{\circ}$ from the major axis (Figs. 1 and 2). Both components - the main body and the suspected ring - are slightly S-shaped.

AM 1934-563 has two nearby companions of similar size and magnitude (Figs. 1 and 2). These are PGC 400092 (NW companion) and PGC 399718 (S companion), both of unknown redshift. Other galaxies of comparable magnitudes are located about $10^{\prime} \mathrm{N}$ of AM 1934-563 and belong to the AM 1934-562 group of galaxies.

AM 1934-563 is an almost unexplored galaxy. Reshetnikov et al. (2001) have found fast rotation of the gas in the central region of the galaxy, as signs of Sy2 or LINER activity. van Driel et al. (2002) have detected 21-cm HI line emission toward AM 1934-563 but the observed HI profile may be confused with another galaxy of the triplet.

We present here new photometric and spectroscopic data for AM 1934-563 and two companion galaxies. The new data 


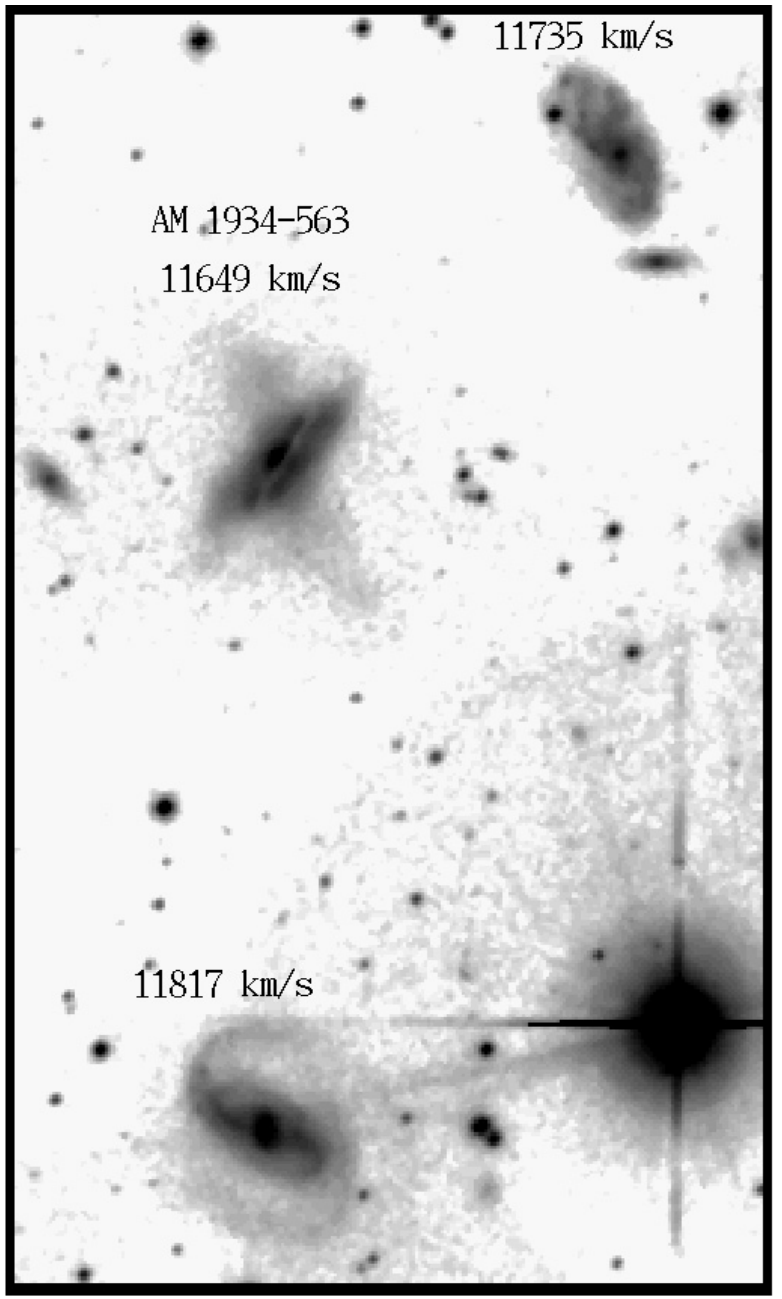

Fig. 1. $V$-band image of AM 1934-563. Image size is $2 ! 1 \times 3$ 3.6. North $^{\prime}$ is at the top, east to the left.

reveal that the three galaxies form a physical triplet. Global observational structure and kinematics of AM 1934-563 allow us to conclude that this galaxy is a real polar-ring galaxy with a spiral host and inclined ring/disk structure.

We also model our observations using N-body numerical simulations. We study different formation scenarios for this polar ring, and find that only one is likely to reproduce the observed properties of AM 1934-563. We conclude that this polar ring has been formed by tidal accretion of material from a gas-rich galaxy, that may still be observed today in the triplet. AM 1934-563, with its massive spiral host, is peculiar among PRGs, which most frequently have a small early-type host (often S0s). Thus, we study the formation of AM 1934-563 in particular, and our results may not apply to PRGs in general, even if many PRGs probably formed through the same accretion mechanism (see $\mathrm{BC} 03$ ).

The observations are presented in Sect. 2. The results are analyzed in Sect. 3. In Sect. 4, we model this system, and discuss its formation mechanism.

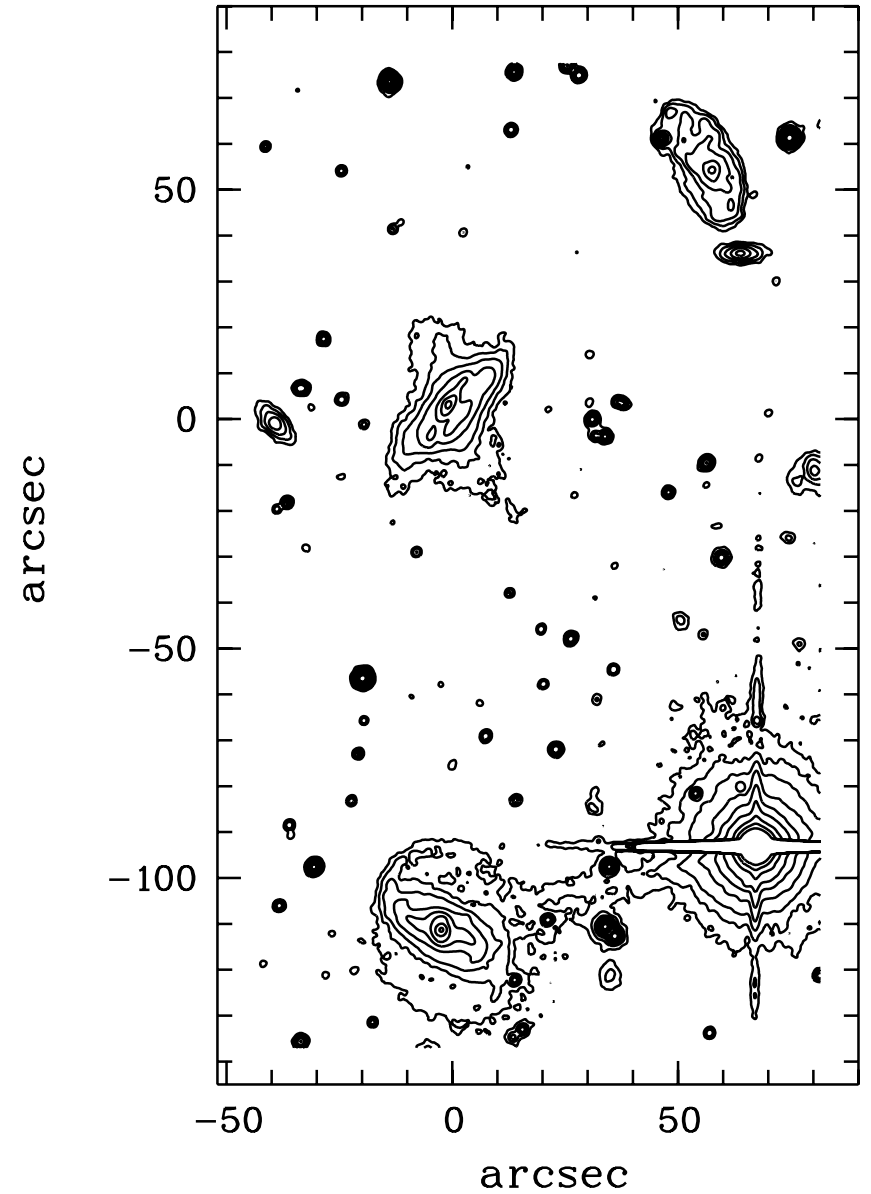

Fig. 2. $V$-band contour map of AM 1934-563. The faintest contour is $24.4 \mathrm{mag} \operatorname{arcsec}^{-2}$, isophotes step -0.75 .

\section{Observations and reductions}

\subsection{Photometric observations}

The photometric observations were performed with the 1.6-m telescope at the OPD (MCT/LNA), Brasil in August 2002. The telescope was equipped with direct imaging camera \#1 and a thick, back-illuminated $2048 \times 2048,13.5-\mu \mathrm{m}$ square pixels CCD detector \#98 (pixel size is 0 ' $18 \times 00^{\prime} 18$ ). The readout noise was $2.4 e^{-}$and the gain, $2.5 e^{-} / \mathrm{ADU}$.

The data were acquired with standard Johnson $B, V$ and Cousins $R, I$ filters. Photometric calibration was accomplished using repeated observations of standard stars from the Landolt (1983) and Graham (1982) lists. The seeing during the observations was 1".3. A log of observations is given in Table 1 . Reduction of the CCD data has been performed in the standard manner using the ESO-MIDAS ${ }^{1}$ package. This includes dark and bias subtraction, flat-field correction (we have used a mean of several dome flats taken in the appropriate filter), sky subtraction. The cosmetic defects and projected stars were excluded by median filtering (for small-scale defects and cosmic rays) or by masking them with rectangular regions. The typical uncertainty of the background level is $(0.4-0.5) \%$. The $I$ band

${ }^{1}$ MIDAS is developed and maintained by the European Southern Observatory. 
Table 1. Observations at OPD.

\begin{tabular}{cccc}
\hline \hline Data & $\begin{array}{c}\text { Band- } \\
\text { pass }\end{array}$ & $\begin{array}{c}\text { Exp } \\
(\mathrm{s})\end{array}$ & $\begin{array}{c}Z \\
\left({ }^{\circ}\right)\end{array}$ \\
\hline 12/13 Aug. 2002 & $B$ & $2 \times 900$ & 35 \\
& $V$ & $3 \times 600$ & 34 \\
& $R$ & $3 \times 600$ & 38 \\
& $I$ & $3 \times 600$ & 35 \\
\hline
\end{tabular}

Table 2. Observations at CTIO.

\begin{tabular}{cccc}
\hline \hline Data & Galaxy & Exp & PA( $\left(^{\circ}\right)$ \\
\hline 27 and 28 Sep. 2002 & AM 1934-563 & $3 \times 1800^{\mathrm{s}}$ & $130^{\circ}$ \\
& AM 1934-563 & $1200^{\mathrm{s}}$ & $27^{\circ}$ \\
& PGC400092 & $2 \times 1200^{\mathrm{s}}$ & $130^{\circ}$ \\
& PGC399718 & $2 \times 1200^{\mathrm{s}}$ & $90^{\circ}$ \\
\hline
\end{tabular}

frames were obtained with insufficient exposure times for the detailed photometry and we used the frames for the integral photometry only.

The total magnitude of AM 1934-563 $(B=15.95 \pm 0.10)$ found by us from the multi-aperture photometry is in good agreement with the $\operatorname{NED}^{2}(B=15.97)$ and $\operatorname{LEDA}^{3}(B=$ $15.93 \pm 0.10)$ data. The two other galaxies show worse agreement: $16.74 \pm 0.10$ vs. $16.17 \pm 0.10$ (LEDA) for the NW companion, and $16.09 \pm 0.11$ vs. $15.74 \pm 0.10$ (LEDA) for the southern galaxy of the triplet. Possible reasons for this discrepancy are the contributions from nearby stars (PGC 400092 and 399718) and from the background galaxy (PGC 400092) (Figs. 1 and 2).

\subsection{Spectral observations}

The spectroscopic observations were performed with the 1.5-m telescope at CTIO in October 27, 2002, equipped with a Cassegrain spectrograph and CCD Loral $1 \mathrm{~K} \# 1$, Arcon 3.9 IRAF software interface, square pixels size $15 \mathrm{mi}$ cron. The grating \#35 of 600 lines $/ \mathrm{mm}$ was centered at $675 \mathrm{~nm}$ (plate scale $18^{\prime \prime} .06 / \mathrm{mm}$, scale slit $11^{\prime \prime} 33 /$ pixel scale dispersion

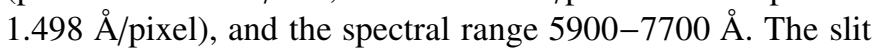
was set at $3^{\prime \prime}$. The seeing during our observations was about $1^{\prime \prime}$. A log of observations is given in Table 2 .

The reductions were carried out with standard techniques using IRAF ${ }^{4}$ and ESO-MIDAS packages. The emission-line kinematics was measured by the Gaussian fit of the brightest emission lines present in the spectra (H $\alpha$ and [NII] 26583$)$. We considered as systemic velocities the values corresponding to the maxima of the continuum intensity. Our derived heliocentric systemic velocity for the AM 1934-563 $\left(11649 \pm 10 \mathrm{~km} \mathrm{~s}^{-1}\right)$ is in agreement with previous optical measurements (see compilation of data in van Driel et al. 2002) but significantly larger than the HI value (van Driel et al. 2002).

\footnotetext{
2 NASA/IPAC Extragalactic Database.

${ }^{3}$ Lyon-Meudon Extragalactic Database.

4 IRAF is distributed by NOAO, which is operated by AURA Inc., under contract with the National Science Foundation.
}

Table 3 summarizes the main observational characteristics of three galaxies. The errors of heliocentric radial velocities represent the formal standard deviations from the averaging of velocities obtained using different lines and spectra. Absolute magnitudes in the table were corrected for the internal absorption according to Tully et al. (1998).

\section{Results and discussion}

\subsection{Dynamical characteristics of the triplet}

AM 1934-563 and two neighboring galaxies show very close radial velocities (Fig. 1, Table 3 ) and very probably form physical triplet. Considering this group as an individual dynamical system, we computed the following standard quantities (see Karachentseva \& Karachentsev 2000 for the corresponding formulae):

- The dispersion of galaxy velocities with respect to the center: $s_{v}=69 \mathrm{~km} \mathrm{~s}^{-1}$.

- The mean harmonic projected distance: $r_{\mathrm{H}}=82.2 \mathrm{kpc}$.

- The dimensionless crossing time of the system: $\tau=0.17$ (in units of the Hubble time, $H_{0}^{-1}$ ).

- The virial mass: $M_{\text {vir }}=1.3 \times 10^{12} M_{\odot}$.

- The virial mass-to-luminosity ratio for the triplet in solar units: $f=14$.

From the comparison of the above characteristics with the corresponding median values of known isolated triplets (Table 3 in Karachentseva \& Karachentsev 2000) we can conclude that the AM 1934-564 system shows about half the size of the $s_{v}$ value. The characteristic size of the triplet $-r_{\mathrm{H}}-$ is close to median values for the southern and northern triplets. The relatively low value of the observed dispersion leads to a large crossing time, and small virial mass and mass-to-luminosity ratio in comparison with typical triplets.

\subsection{Nuclear spectra of the galaxies}

The nuclear $\mathrm{H} \alpha$ spectra of galaxies are presented in Fig. 3. The results of our measurements of the nuclear emission-line properties within $4^{\prime \prime} \times 3^{\prime \prime}$ are summarized in Table 4 .

The NW member of the triplet shows typical HII regionlike spectrum. AM 1934-563 demonstrates a relatively large [NII] $\lambda 6583 / \mathrm{H} \alpha$ ratio and noticeable width of the emission lines, but the galaxy shows a comparatively faint $[\mathrm{OI}] \lambda 6300$ line $([\mathrm{OI}] \lambda 6300 / \mathrm{H} \alpha \leq 0.05)$. Therefore, we can classify its spectrum as a transition - AGN/HII - type (Veilleux \& Osterbrock 1987).

Emission lines in the nuclear spectrum of PGC 399718 (S galaxy) are double-peaked and wide (Fig. 3), reflecting very steep rotation curve visible in the two-dimensional spectrum. (The atlas by Ho et al. (1995) gives several examples of such objects - e.g., NGC 488, NGC 3245, and NGC 4772.) The amplitude of splitting determined from the Gauss decomposition of the $\mathrm{H} \alpha$ and [NII] $\lambda 6583$ contours is $250-280 \mathrm{~km} \mathrm{~s}^{-1}$. 
Table 3. General properties of the galaxies.

\begin{tabular}{|c|c|c|c|c|}
\hline Parameter & AM 1934-563 & PGC $400092(\mathrm{NW})$ & PGC $399718(\mathrm{~S})$ & Ref. \\
\hline$\alpha(2000)$ & $19^{\mathrm{h}} 38^{\mathrm{m}} 38^{\mathrm{s}} .52$ & $19^{\mathrm{h}} 38^{\mathrm{m}} 31^{\mathrm{s}} .55$ & $19^{\mathrm{h}} 38^{\mathrm{m}} 38^{\mathrm{s}} \cdot 69$ & \\
\hline$\delta(2000)$ & $-56^{\circ} 27^{\prime} 28^{\prime \prime} .5$ & $-56^{\circ} 26^{\prime} 36^{\prime \prime} .7$ & $-56^{\circ} 29^{\prime} 22^{\prime \prime} .3$ & \\
\hline Morphological type & $\mathrm{SBa} / \mathrm{b}:$ & $\mathrm{Sd} / \mathrm{Irr}:$ & SBc: & \\
\hline Heliocentric systemic velocity* $\left(\mathrm{km} \mathrm{s}^{-1}\right)$ & $11649 \pm 10$ & $11735 \pm 6$ & $11817 \pm 26$ & \\
\hline Adopted luminosity distance ${ }^{* *}$ & & 167.6 Mpc & & \\
\hline Scale & & $0.75 \mathrm{kpc} / 1^{\prime \prime}$ & & \\
\hline Major axis, $\mathrm{D}_{26}\left(\mu_{B}=26\right)$ & $50^{\prime \prime}(38 \mathrm{kpc})$ & $36^{\prime \prime}(27 \mathrm{kpc})$ & $43^{\prime \prime}(32 \mathrm{kpc})$ & \\
\hline Axial ratio & $0.45:$ & 0.40 & $0.6:$ & \\
\hline \multicolumn{5}{|l|}{$\begin{array}{l}\text { Total apparent } \\
\text { magnitudes and colors: }\end{array}$} \\
\hline$B_{\mathrm{T}}$ & $15.95 \pm 0.10$ & $16.74 \pm 0.10$ & $16.09 \pm 0.11$ & \\
\hline$(B-V)_{\mathrm{T}}$ & $+0.94 \pm 0.04$ & $+0.61 \pm 0.04$ & $+1.15 \pm 0.05$ & \\
\hline$(V-R)_{\mathrm{T}}$ & $+0.60 \pm 0.03$ & $+0.38 \pm 0.03$ & $+0.45 \pm 0.05$ & \\
\hline$(R-I)_{\mathrm{T}}$ & $+0.51 \pm 0.1$ & $+0.2 \pm 0.1$ & $+0.35 \pm 0.1$ & \\
\hline$(J-H)_{2 \text { MASS }}$ & +0.83 & & +0.57 & {$[1]$} \\
\hline$(H-K)_{2 \mathrm{MASS}}$ & +0.44 & & +0.29 & [1] \\
\hline Galactic absorption ( $B$-band) & 0.22 & 0.22 & 0.22 & {$[2]$} \\
\hline K-correction $(B$-band $)$ & 0.07 & 0.01 & 0.05 & [3] \\
\hline Absolute magnitude, $M_{B}^{0}$ & -21.1 & -20.2 & -20.7 & \\
\hline$L_{\mathrm{FIR}}\left(L_{\odot}\right)$ & $8.8 \times 10^{10}$ & & & NED \\
\hline $\operatorname{SFR}_{F I R}\left(M_{\odot} / \mathrm{yr}\right)$ & 45 & & & \\
\hline
\end{tabular}

* - conventional radial velocity obtained as $c z$ (Fairall 1992).

${ }^{* *}-\Omega_{\mathrm{m}}=0.3, \Omega_{\Lambda}=0.7, H_{0}=70 \mathrm{~km} \mathrm{~s}^{-1} / \mathrm{Mpc}$.

[1] - Skrutskie et al. (1997); [2] - Schlegel et al. (1998); [3] - Bicker et al. (2004).

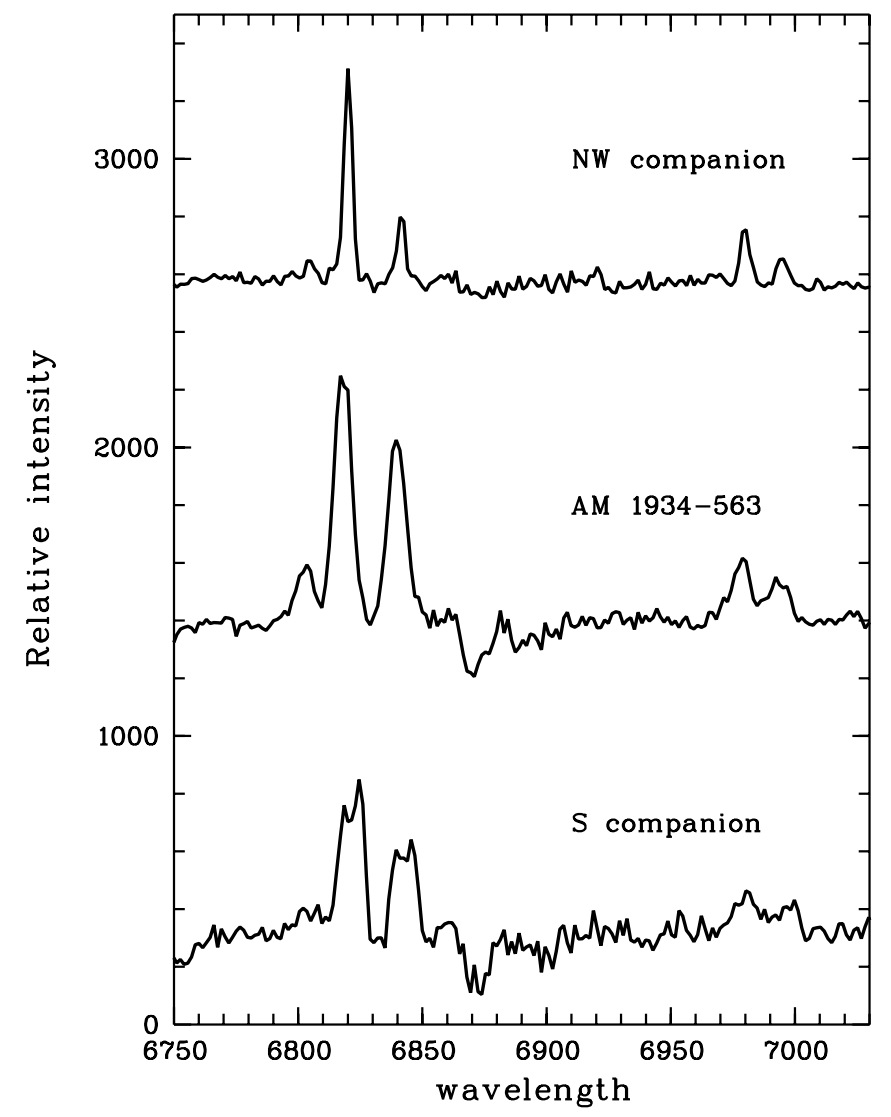

Fig. 3. Arbitrary scaled nuclear $\mathrm{H} \alpha$ spectra of the triplet members.

\section{3. $A M$ 1934-563}

\subsubsection{Main galaxy}

The main object is a distorted edge-on spiral galaxy (Figs. 1 and 2). The outer parts of the galaxy show significant warp of the isophotes with amplitude of $\sim 8^{\circ}$. The bright inner part of the disk with PA $=148^{\circ}$ is crossed by a prominent dust lane and is inclined with respect to the outer isophotes by about $13^{\circ}$. This inner inclined structure could represent an almost edge-on bar. Detailed inspection shows that the dust lane is split and embraces the galaxy nucleus from SE and NW.

Figure 4 displays the surface brightness profiles of AM 1934-563 approximately along the major axis and along the bright inner part of the outer structure (see Sect. 3.3.2). The surface brightness was averaged within $5 \times 5$ pixels or 0 ".9 $\times 0$ ".9. The major axis profile shows a central peak and two depressions at $|r| \approx 5^{\prime \prime}$ probably related to the dust lanes. At $|r| \geq 10^{\prime \prime}$ the major axis profile can be approximated by an exponential disk with a central surface brightness $\mu_{0}(B)=20.06 \pm 0.19$ (corrected for the Milky Way extinction and K-correction) and exponential scale-length $h(B)=$ $5{ }^{\prime \prime} 1 \pm 0.3$ (3.8 kpc). Therefore, the disk of AM 1934-563 is typical for high surface brightness, star-forming disks of other known PRGs with spiral hosts (Reshetnikov 2004).

The disk of AM 1934-563 shows strong color gradients (Fig. $4 \mathrm{~b})$ : central parts of the galaxy are red $(B-V \approx+1.1$, $V-R \approx+0.9)$, while the outer ones are blue $(B-V \approx+0.6$, 

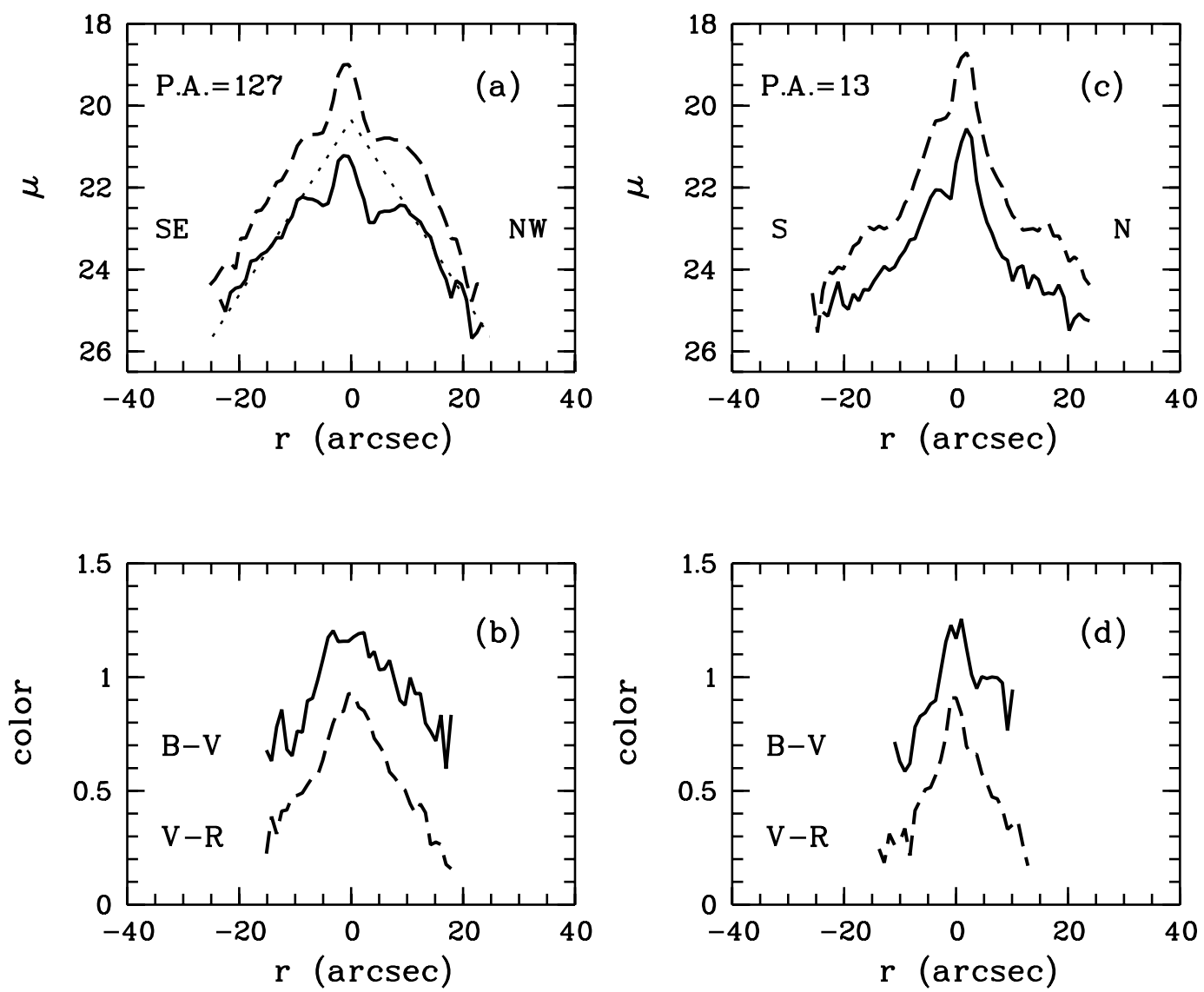

Fig. 4. Photometric profiles for AM 1934-563: a), b) along the apparent major axis; c), d) along the major axis of the suspected ring. Solid line in a) and c) represent the distributions in the $B$ passband, dashed lines in $R$. Dotted line in a) shows the symmetric exponential model for the disk.

Table 4. Nuclear emission-line properties.

\begin{tabular}{cccc}
\hline \hline Parameter & AM 1934-563 & NW galaxy & S galaxy \\
\hline$F W H M(\mathrm{H} \alpha), \mathrm{km} \mathrm{s}^{-1}$ & $320 \pm 20$ & $<100$ & $440 \pm 40$ \\
$W_{\lambda}(\mathrm{H} \alpha), \AA$ & $12.1 \pm 0.7$ & $17.5 \pm 0.6$ & $9.1 \pm 0.8$ \\
{$[\mathrm{NII}] \lambda 6583 / \mathrm{H} \alpha$} & $0.74 \pm 0.04$ & $0.32 \pm 0.08$ & $0.6 \pm 0.1$ \\
{$[\mathrm{SII}] / \mathrm{H} \alpha$} & $0.33 \pm 0.1$ & $0.45 \pm 0.1$ & $0.25 \pm 0.1$ \\
\hline
\end{tabular}

$V-R \approx+0.3)$. The observed ratios of the scale-lengths in different color bands are $h(B) / h(V)=1.18 \pm 0.11, h(B) / h(R)=$ $1.25 \pm 0.12$. Such color gradients are very common among faceon (e.g., de Jong 1996) and edge-on (e.g., de Grijs 1998) spiral galaxies. De Grijs (1998) argues that the observed radial color gradients in edge-on galaxies largely represent their dust content.

The optical colors of the galaxy corrected for the Milky Way absorption, K-correction and rather uncertain internal absorption are $(B-V)_{0} \approx 0.6-0.7$ and $(V-R)_{0} \approx 0.4-0.5$. These values are usual for the $\mathrm{Sa}-\mathrm{Sb}$ galaxies (Buta et al. 1994; Buta \& Williams 1995).

AM 1934-563 is a strong source of far-infrared emission (PRC, Table 3). Converting the far-infrared luminosity to the star formation rate (Bushouse 1987), we obtain $45 M_{\odot} / \mathrm{yr}$ and $L_{\mathrm{FIR}} / L_{B}=3.4$. Both estimates are very high in comparison with the data for isolated and even for interacting galaxies (e.g.,
Bushouse 1987). We suppose that the observed IRAS flux can be contaminated by the neighboring members of the triplet. Even in the case where we divide the SFR and $L_{\mathrm{FIR}} / L_{B}$ estimates by 3 (roughly), AM 1934-563 is a galaxy with very active star formation.

Figure 5 shows the emission-line rotation curve of the galaxy (after correction for the cosmological stretch). Thin solid line in the bottom panel is a fit by an exponential disk with $h(R)=4$ "' $1(3.1 \mathrm{kpc})$ and intrinsic axial ratio 0.2 (Monnet \& Simien 1977). It can be seen that the exponential disk approximation gives a good description of the observed rotation curve within $12^{\prime \prime}(9 \mathrm{kpc})$ from the nucleus. The maximum rotation velocity of the galaxy obtained is $V_{\max }=195 \mathrm{~km} \mathrm{~s}^{-1}$. The actual $V_{\max }$ value can be somewhat larger due to peculiar appearance of the galaxy and uncertain inclination. The Tully-Fisher relation predicts that the extinction-corrected luminosity of a galaxy with $V_{\max }=195 \mathrm{~km} \mathrm{~s}^{-1}$ is $M(B) \approx-21$ (Tully et al. 1998; Kannappan et al. 2002). Therefore, AM 1934-563 equatorial disk lies close to the Tully-Fisher relation for normal spirals (at least, in the first order approximation).

Assuming a spherical mass distribution and a flat rotation curve within the optical radius $\left(R_{26}=19 \mathrm{kpc}\right)$, we can estimate the AM 1934-563 mass as $1.68 \times 10^{11} M_{\odot}$. Therefore, the massto-luminosity ratio in the $B$ passband is 4 in solar units - a rather normal value for a giant spiral galaxy. 

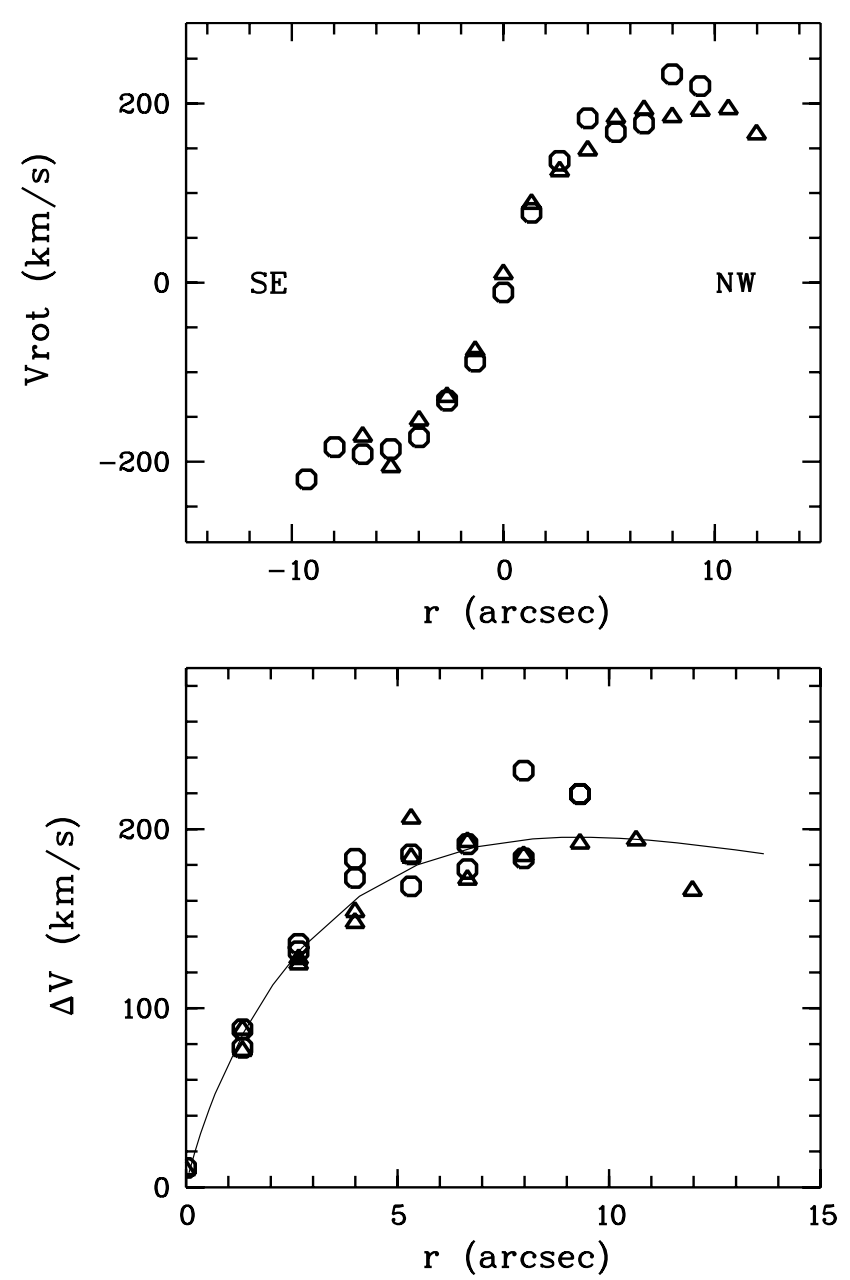

Fig. 5. Top - radial velocities distribution along the major axis of AM 1934-563 $\left(\mathrm{PA}=130^{\circ}\right)$, bottom - averaged rotation curve (circles - H $\alpha$ data, triangles - [NII] 65583 ). Thin solid line represents the rotation curve of an exponential disk with scalelength of 4.'1 (scalelength value in the $R$ filter).

\subsubsection{Possible polar ring}

Figure 6 presents a deep contour map of AM 1934-563. The most fascinating feature of the galaxy is a giant (optical diameter reaches $60^{\prime \prime}$ or $45 \mathrm{kpc}$ ) inclined S-shaped structure which crosses the main body. From the morphological point of view, the off-plane structure resembles the polar rings or disks of the polar-ring galaxies (see examples in the PRC). The appearance of AM 1934-563 is very similar to NGC 660 (see Fig. 1 in van Driel et al. 1995). But, in contrast to NGC 660 which is a sub- $L^{*}$ galaxy, AM 1934-563 is a giant spiral galaxy.

The ring is very faint. Even the brightest inner parts show $\mu(B) \approx 24$ (Fig. $4 \mathrm{c}$ ). As the main galaxy, the ring shows a color gradient (Fig. 4d).

The polar ring (or polar disk) shows a strong warp. Its inner part is inclined about $57^{\circ}$ with respect to the AM 1934-563 major axis and the outer one is inclined about $72^{\circ}$.

The ring is asymmetric. It extends up to $26^{\prime \prime}$ to the NE from the nucleus, and up to $34^{\prime \prime}$ in the opposite direction. The color distribution along the ring is also asymmetric: the NE (shorter)

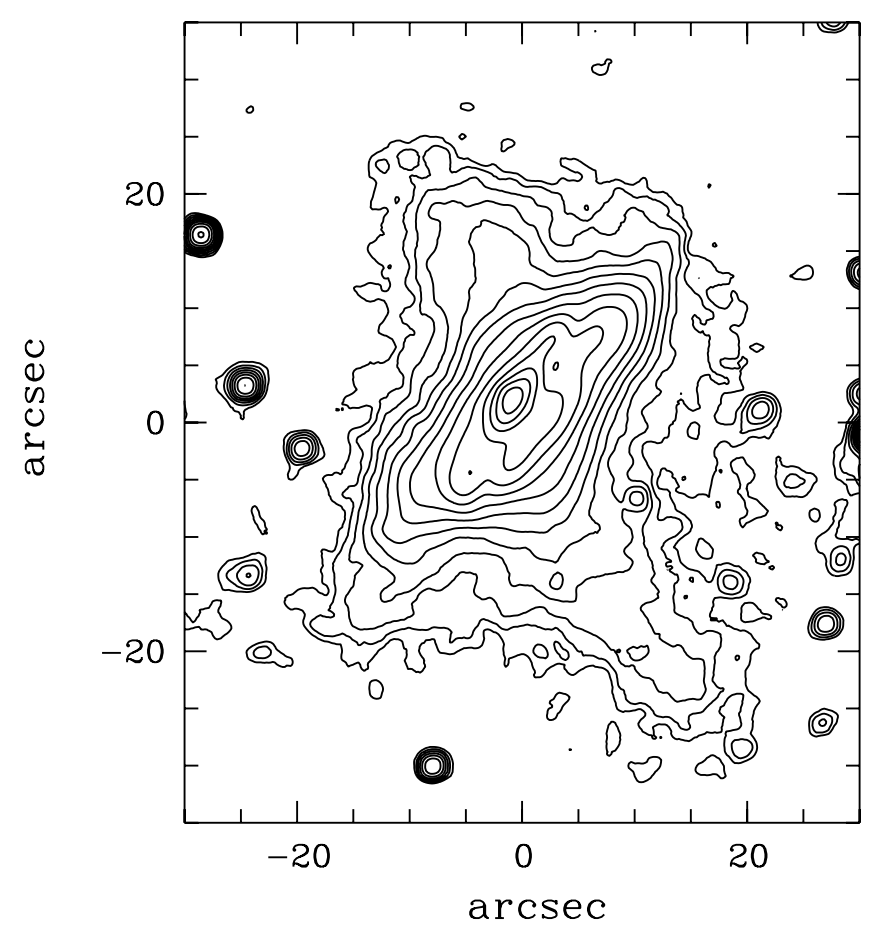

Fig. 6. Contour map of AM 1934-563 from the combined $B+V+R$ image. Isophotes are separated by factor 1.5 .

part of the ring looks redder in the $B-V$ (Fig. 4d) and this color asymmetry can be traced up to the end of the ring.

The observed colors of the ring are relatively blue in comparison with the colors of central galaxy. Its mean colors corrected for the Milky Way extinction and K-correction are $B-V=0.7, V-R=0.35$ (ring colors were measured over 6 .' 5 diameter apertures placed at $15^{\prime \prime}-25^{\prime \prime}$ on the both sides of the nucleus of the galaxy). Taking into account that the ring is almost edge-on, its real "face-on" colors may be significantly bluer.

The total observational luminosity of the ring is about (10-15)\% of AM 1934-563. Therefore, the rings absolute luminosity can reach $M(B) \approx-18.5--19$. So the AM 1934-563 ring is comparable with the brightest ring structures among nearby PRGs (e.g., Reshetnikov et al. 1994).

Figure 7 shows the distribution of radial velocities along $\mathrm{PA}=27^{\circ}$. Unfortunately, the slit goes through the faint periphery regions of the ring but misses the bright inner ones. The forbidden [NII] $\lambda 6583$ line demonstrates almost constant velocity close to the galaxy systemic velocity. The $\mathrm{H} \alpha$ line gives only marginal evidence for the velocity gradient along the ring (the dotted line in Fig. 7 shows linear fit of the data). Interestingly, the direction of this gradient coincides with the direction of the main body rotation (Fig. 5).

Along $\mathrm{PA}=27^{\circ}$ we observe the rise of the $[\mathrm{NII}] / \mathrm{H} \alpha$ ratio from $0.7-0.8$ in the nucleus to $\sim 1.3$ at $|r|=3^{\prime \prime}-4^{\prime \prime}$. Similar increases in the $[\mathrm{NII}] / \mathrm{H} \alpha$ ratio have been observed recently in a number of edge-on spirals, e.g., UGC 10043 (Matthews $\&$ de Grijs 2004, and references therein). Shock-heating due to starburst-driven wind is the usual explanation of such line ratios. In the case of AM 1934-563 we need additional spectral observations to confirm the presence of a large-scale wind. 


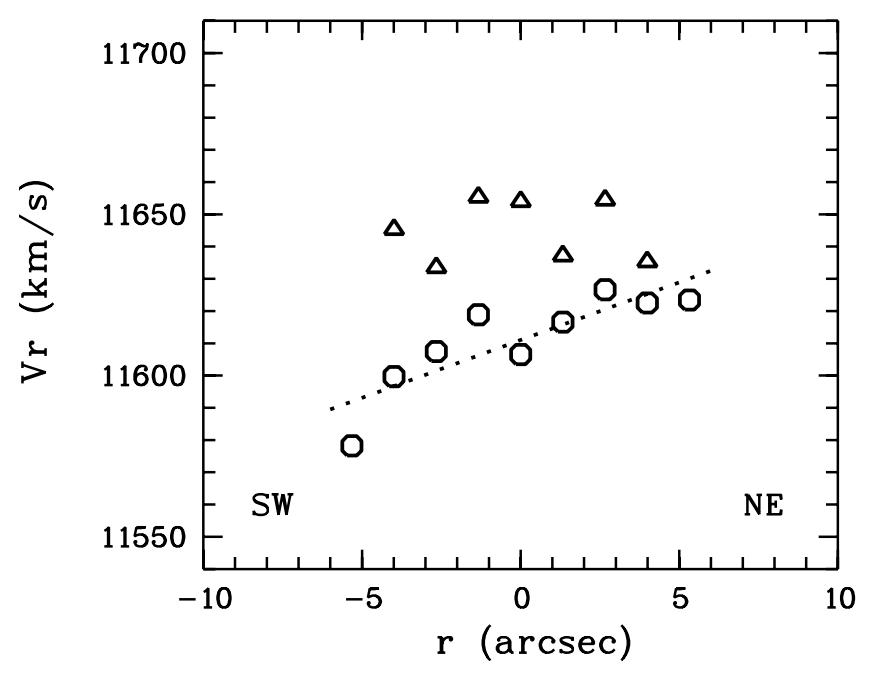

Fig. 7. Radial velocities distribution along the major axis of the ring of AM 1934-563 (circles - H $\alpha$ data, triangles - [NII] $\lambda 6583$ ).

Two other PRGs with young or forming rings are classified as "superwind" galaxies - NGC 660 (Armus et al. 1990) and NGC 6286 (Shalyapina et al. 2004). The possible polar-ring related spiral UGC 10043 also shows the presence of a largescale wind (Matthews \& de Grijs 2004). Nuclear starburst and, correspondingly, starburst-driven wind extending up to the outer kinematically decoupled structure may be the natural consequences of an external accretion event, as suggested by numerical models (BC03, and the model in this paper).

A systematic shift in radial velocities derived from $\mathrm{H} \alpha$ and [NII] lines is evident in Fig. 7. It is not unique feature since many spirals show the same behavior (e.g., Afanasiev et al. 2001; Moiseev 2002). The difference in observed velocities may be explained by the simple assumption that the [NII] emission line originates partially in shocks, due to a wind or a bar (Afanasiev et al. 2001).

\subsection{Companion galaxies}

Both companion galaxies are giant spirals. The NW galaxy (PGC 400092) demonstrates asymmetric, slightly irregular morphology without pronounced spiral arms. The optical colors of the galaxy (corrected for the Milky Way absorption and $\mathrm{K}$-correction) are rather blue $(B-V=0.55, V-R=0.35)$ and are typical for a late-type or irregular galaxy. A small edgeon (may be background) galaxy with $B=19.5, B-V=0.9$, $V-R=+0.6$ (observational values) is located $19^{\prime \prime} \mathrm{SW}$ of the PGC 400092 nucleus.

The southern galaxy of the triplet (PGC 399718) possess a bar and a two-arm spiral structure. The $B-V$ color of the galaxy looks unusually red for spirals and this can be an indication of a high inclination. A high velocity gradient at PA $=90^{\circ}$ (see Sect. 3.2) also supports this point of view. Therefore, the galaxy structure may be not planar - with an almost edge-on inner part and more face-on outer ones.

\section{Modeling of the AM 1934-563 morphology}

\subsection{Numerical techniques}

The simulations were performed with an $N$-body particle code which includes gas dynamics and star formation (see BC03). The main characteristics of this code is that the potential is computed by FFT on a 3D Cartesian grid. The size of the grid is $128^{3}$, and the Fourier images are suppressed by the method of James (1977). The softening length is equal to the size of a grid cell, which is of the order of 1 to $3 \mathrm{kpc}$, according to the models. The particles, stars, gas and dark matter are between 2 and $6 \times 10^{5}$. The gas dynamics is modeled by the sticky-particles scheme.

\subsection{Results}

There are too many free parameters in a numerical model to fit the morphology of AM 1934-563 in detail (see discussion in BC03). Therefore, we decided to find a solution which reasonably describes only the general observational properties of the galaxy. We have considered two scenarios for the polar ring formation: 1) a minor merger with a small companion, where the ring is formed by the tidal disruption of the smallest galaxy and 2) tidal accretion of matter from a massive, gas rich donor galaxy to the polar ring (as in $\mathrm{BC} 03$ ). The major merger scenario (see Introduction) has not been simulated, but models of this scenario can be found in Bekki (1998) and BC03.

Minor merger In the framework of the first scenario, we have considered a minor merger with an inclined orbit, where the ring results from the disruption of the companion. In the present case, the mass of the companion should be $10-20 \%$ of the mass of the host galaxy: more massive companions would disturb the host galaxy too much and form a S0-like object (Bournaud et al. 2004, 2005a), less massive ones can only form rings that are much fainter than in the observed system. On the basis of our observations, the main galaxy stellar mass was set to $M_{\text {stars }}=2 \times 10^{11} M_{\odot}$. The gas fraction was fixed as $M_{\text {gas }} / M_{\text {stars }}=0.2$. We assumed a spherical dark matter halo, described by a Plummer sphere of scale-length $30 \mathrm{kpc}$ and truncation radius $60 \mathrm{kpc}$, with a dark-to-visible mass ratio $M_{\mathrm{DH}} / M_{\text {stars }}=0.6$ inside the stellar radius. This mass was chosen to fit the rotation curve amplitude. The characteristic size of the exponential disk is taken from the surface brightness fit.

We have simulated several minor mergers with a companion mass of $15 \%$ the primary mass. Such a mass ratio is required to form a massive enough ring, without disturbing the host disk more than in the observed case. The internal characteristics of the companion are fixed as $M_{\mathrm{gas}} / M_{\text {stars }}=0.25$ and $M_{\mathrm{DH}} / M_{\text {stars+gas }}=1.5$, and orbital parameters given in Table 5 . The result of two representative simulations is shown in Fig. 8. We found that a minor merger with such a mass ratio can only form unclosed ring-like inclined features:

- for a ring inclined by at least 25 degrees from the polar axis (as in the observed system), differential precession has affected the orbit of the progenitor companion, which results 

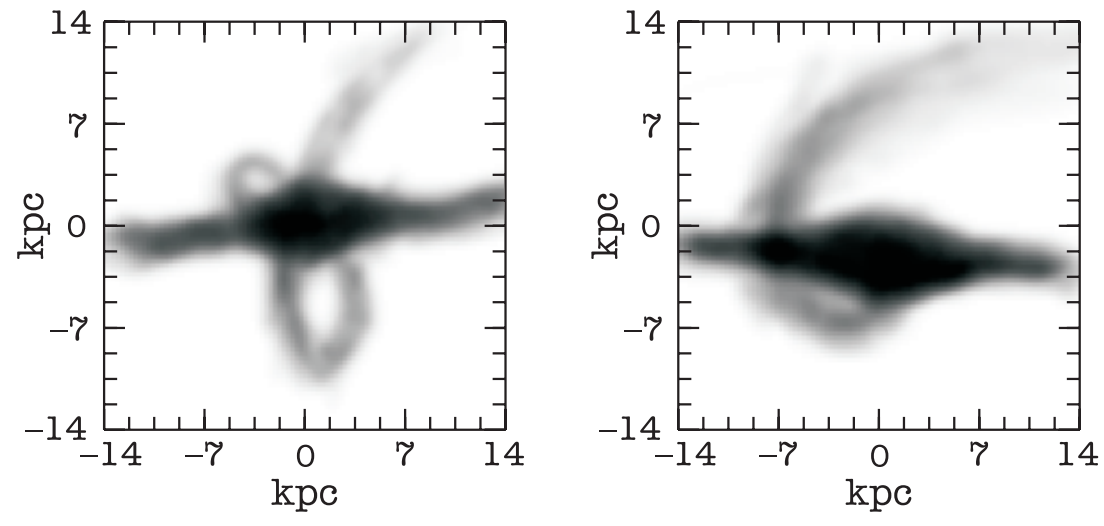

Fig. 8. Projection of the polar ring in two simulations of minor mergers, seen $300 \mathrm{Myr}$ after the merging of the companion (left: run1, initial orbital plane seen close to edge-on - right: run2, initial orbital plane seen face-on). The three aspects making the ring "unclosed" are illustrated by these figures: the orbital plane is largely tilted in one rotation (left), the ring makes less than one rotation (right), and there is a high decay in the radius between both sides of the ring (left and right). Quantitative results for more simulations are given in Table 5. We used a non-linear scale adjusted to enhance the visibility of the ring-like feature.
Table 5. Run parameters and results of simulations of minor mergers, where the disruption of the companion forms a ring-like feature. We indicate the orbital parameters (Cols. 2-4): relative velocity, inclination of the orbital plane w.r.t. a polar plane, and impact parameter. We also give estimates of three characteristics of the ring, showing its unclosed aspect: the number of rotations visible in the ring-like structure, the tilt of the ring plane between the two sides of the ring (related to the precession of the companion orbit), and the ratio of the extend of both sides of the ring (related to the orbital decay of the companion). The tilt of the ring orientation could not be estimated in two cases, because the ring-like structure was not complete enough. These values have been estimated about $300 \mathrm{Myr}$ after the merging of the companion.

\begin{tabular}{ccccccc}
\hline \hline$N$ & $V_{\infty}\left(\mathrm{km} \mathrm{s}^{-1}\right)$ & $\Theta\left({ }^{\circ}\right)$ & $R_{\mathrm{p}}(\mathrm{kpc})$ & rotations & tilt & $R_{1} / R_{2}$ \\
\hline 1 & 250 & 25 & 80 & 1.4 & 40 & 2.5 \\
2 & 100 & 25 & 50 & 0.5 & - & 3.5 \\
3 & 250 & 25 & 50 & 1.3 & 30 & 2.2 \\
4 & 100 & 25 & 80 & 0.8 & 25 & 1.9 \\
5 & 150 & 25 & 120 & 0.9 & 20 & 2.2 \\
6 & 150 & 25 & 40 & 0.6 & - & 2.5 \\
7 & 150 & 0 & 80 & 0.8 & 0 & 2.0 \\
8 & 150 & 35 & 80 & 0.8 & 35 & 2.3 \\
9 & 250 & 35 & 80 & 1.2 & 45 & 2.8 \\
\hline
\end{tabular}

in a significant tilt between both sides of the ring (visible when the ring is seen close to edge-on, e.g. in Fig. 8);

- debris from the companion can often be detected in less than one rotation, making the ring incomplete, in particular when the impact parameter or relative velocity of the companion are small. This is mainly visible when the ring is seen face-on, but for edge-on projections it results in a large discrepancy in the luminosity of both sides of the ring (one side of the projected ring can be several times more luminous than the other one);

- because of the orbital decay of the companion, the two sides of the ring have very different sizes (one side is generally more extended than the other one by a factor of 2-3).

These three aspects are described quantitatively in Table 5 for several simulations of minor mergers. The two sides of the ring of AM 1934-563 have comparable sizes, comparable masses, and form a continuous structure with a rather constant orientation. This cannot be reproduced by simulations of minor mergers, for an inclined ring. In particular the discrepancies in the mass/extent of both sides of the observed ring are much lower than in simulations of minor mergers. The general problem of this scenario is that a companion on an inclined orbit does not have enough time to stretch more or less uniformly along the orbit before merging with the primary, because the time-scale of dynamical friction (that has a typical time-scale of $0.55 \mathrm{Gyr}$ in our simulations) is not much larger than the dynamical timescale (that has an average value of $0.4 \mathrm{Gyr}$ ). Thus, differential precession and radial decay affect the orbit faster than the material is captured into the ring, which does not allow the morphology of a closed ring to be reproduced. It thus seems unlikely that the observed polar ring is the result of a minor merger. Moreover, these unclosed ring-like structures are generally short lived, and are dispersed by differential precession in less than $1 \mathrm{Gyr}$. With a more massive companion, they could be longer-lived, because self-gravity would reduce the effects of differential precession, but such a companion would make the host galaxy become an extremely early-type disk galaxy, or even an S0 (see Bournaud et al. 2004, 2005a), which is not observed.

We have not varied the dark halo density profile. This could be an important parameter, because it changes the timescale for dynamical friction, which can affect the ring being closed or not. However, we have widely varied the velocity and impact parameter of the companion. Then, the disruption of the companion occurs at various radii, around various densities of dark matter, thus under different degrees of dynamical friction. This change does not allow a closed ring to be formed. Changing the halo profile would change the dynamical friction over a similar range, so the conclusion would be the same, namely that it cannot explain the formation of the ring of AM 1934-563.

We conclude that the observed polar ring is not the result of the stripping of a small companion during a minor merger.

Tidal accretion In a second scenario we have considered an unbound encounter of the primary (target) with a gas rich giant spiral galaxy (donor). Tidal mass transfer from the donor to the target is then assumed to form a polar ring around the target. The mass of the target galaxy is $2 \times 10^{11} M_{\odot}$. In the best fitting model that we have found, the donor galaxy has a stellar disk of radius $17 \mathrm{kpc}$, and its gaseous disk extends up to $42 \mathrm{kpc}$. Its visible mass is $3.6 \times 10^{11} M_{\odot}$, and the mass fraction of gas within its visible matter, including gas outside the stellar radius, is 0.33 . The target galaxy has a stellar radius of $14 \mathrm{kpc}$. 
It contains a gas fraction $M_{\mathrm{gas}} / M_{\mathrm{stars}}=0.18$, with no gas outside its stellar disk, as is the case for many early-type galaxies. Both galaxies are embedded in a dark halo described by a Plummer sphere of radial scale-length $30 \mathrm{kpc}$ and truncation radius of $60 \mathrm{kpc}$. The dark-to-visible mass ratio inside the stellar disk radius is 0.6 for the target galaxy, and 0.5 for the donor galaxy. The initial conditions of the encounter have to be selected carefully in order to favor inclined accretion of the tidal tail. The most suitable conditions we have found are: impact parameter $130 \mathrm{kpc}$, relative velocity of $V=145 \mathrm{~km} \mathrm{~s}^{-1}$, almost perpendicular orientation of the donor disk and the orbital plane with respect to the host galaxy disk - but not exactly perpendicular, so that the ring is both inclined and warped. The simulation produced an inclined, large-scale, faint stellar ring around the main galaxy. If we denote $t=0$ as the beginning of the simulation, the pericenter passage occurs at $t=780 \mathrm{Myr}$. A tidal bridge is formed between the two galaxies, and is accreted to a polar structure (Fig. 9). The polar ring is first open and irregular, but becomes closed and regular in a few $10^{8} \mathrm{yr}$, matching the observed appearance of AM 1934-563. While rings resulting from minor-merging companions appear to remain unclosed in our simulations, the ring is here unclosed only for a short time, when it is an unrelaxed, still forming structure. Even less than $1 \mathrm{Gyr}$ after its formation the ring is likely to be observed as a closed structure. Thus, that the ring of AM 1934563 is closed is not a strong constraint for the scenario of tidal accretion from a massive donor. The reason is that the ring material is captured by the host galaxy over about $0.3 \mathrm{Gyr}$, faster than the dynamical friction timescale, which allows the ring to be closed. This is the contrary of the minor merger scenario, where the material is captured over more than one rotation of the companion, i.e. at least $0.5 \mathrm{Gyr}$ : in this other case we have shown that the ring does not become closed.

Figure 10 presents contour plots of stars in the simulated polar-ring galaxy at $t=1600$ Myrs (820 Myrs after the pericenter passage). At that time the donor galaxy has moved away by about $250 \mathrm{kpc}$ (which can appear much closer in the sky projection) and does not perturb the target any longer; the polar ring has become more regular than in the earlier stages shown in Fig. 9, even if still asymmetrical. This model reproduces the main characteristics of AM 1934-563:

- the polar ring inclination, and the relative sizes of the polar ring and the host galaxy, are well reproduced. The projected distribution of stars along the major axis of the model galaxy is well approximated by an exponential disk with $h=2.8 \mathrm{kpc}$ (for AM 1934-563 we have found $h=3.1 \mathrm{kpc}$ );

- the model fits the warp of the polar ring, and the S-shape of the host galaxy. The latter is caused by a small warp but also a barred-spiral structure, since the host disk is not observed perfectly edge-on;

- the asymmetric appearance of the ring, with its southern part more extended and less luminous than the northern one, is reproduced;

- in the model, the contribution of the ring to the total luminosity is about $11 \%$. This percentage agrees reasonably with the observational value (Sect. 3.3.2): the mass of the polar structure is comparable to the mass of the observed polar ring.

The matter transferred from the donor to the target galaxy is not only gas. As can be seen in Fig. 9, some stars from the donor are also captured in the polar disk, without being dispersed, but most of them were formed just before the ring, inside the tidal bridge. That some stars are formed before the ring may lead to an over-estimation of the age of the ring, yet these stars do not dominate the mass and are not much older than the ring, so that this over-estimation does not exceed $1-2 \times 10^{8} \mathrm{yr}$. A small fraction of dark matter is also accreted from the donnor galaxy, but for our model with non-rotating dark haloes, this does not exceed $5 \%$ of the donnor dark mass.

\subsection{Formation scenarios}

At least four scenarios can be proposed to account for young, massive polar rings surrounding pre-existing host galaxies:

- the major merger scenario (e.g., Bekki 1998), where the two merging protagonists are found with one in the host, and the other in the polar ring: the polar ring is the remnant of a pre-existing disk;

- the disruption of a small companion on a polar orbit, the debris of which forms the polar ring;

- tidal transfer of material from a massive donor during a galaxy encounter. The donor is not necessarily merging with the host galaxy, and keeps its identity after the event;

- accretion of gas infalling from cosmic filaments towards the polar structure. Gas can be accreted gradually and form a purely gaseous polar ring in a first step, then an external perturbation triggers star formation in the ring, as proposed by Cox et al. (2001) for the system on interacting galaxies II Zw 70/71.

The last three scenarios can be regarded as three subtypes of the accretion scenario. The first and third of these variants do not predict the presence of companions around PRGs, which is supported by observations. For example, Brocca et al. (1997) find that statistically, the environments of PRGs show no excess of close companions, which is consistent with the majority of PRGs forming via either long-term secular gas accretion or via mergers in which the companion is destroyed. However, this does not rule out accretion from a gas-rich donor as the donor may have migrated away. Iodice et al. (2002) favor the merger scenario of two disk galaxies from the study of stellar populations and morphological structure of the host; however, the constraints are not strong enough, since in any scenario, gas can be accreted by the host and induce star formation, while the host is perturbed, so that a large range of stellar ages should coexist in the perturbed host. The presence of a diffuse envelope of stars around the PRG is more constraining (see BC03).

The major merger scenario has been shown to be less likely than the tidal accretion scenario, especially for inclined rings (BC03). Moreover, the asymmetric ring of AM 1934-563 cannot be well fitted by this scenario. Indeed, this scenario assumes that the host galaxy has merged with a "victim" disk galaxy that has given birth to the polar ring. Since the southern part of 

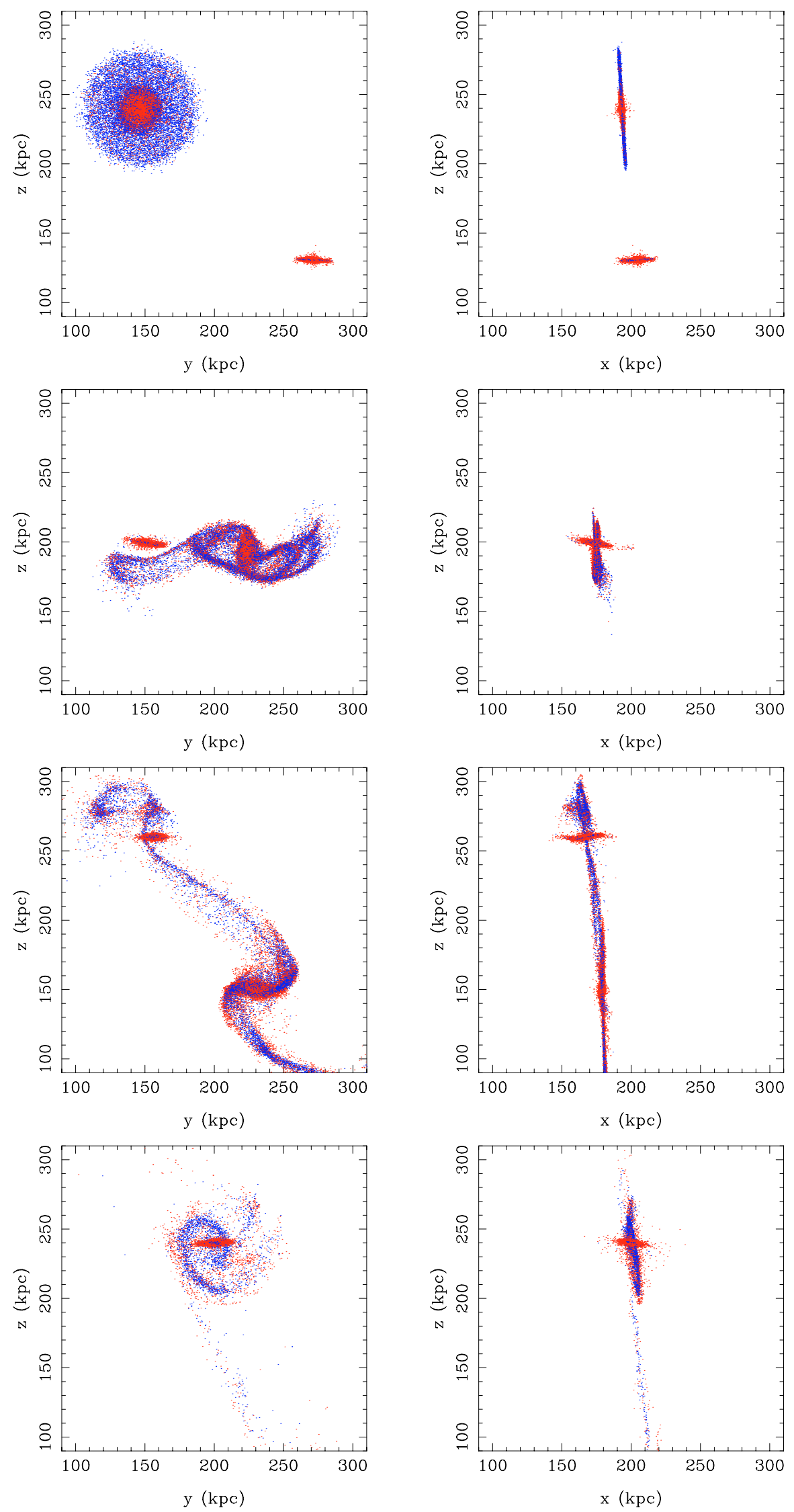

Fig. 9. Two projections of the polar-ring formation run (two columns), seen at $t=200,700,900$, and 1150 Myr. Stellar particles are in red, gas clouds in blue (30000 particles are plotted). In the last snapshot, the ring has just formed and is not closed yet. It is closed and more regular a few $10^{8}$ Myr later, as shown in Fig. 10, while closed rings are not formed in our simulations of disruption of a minor companion. 

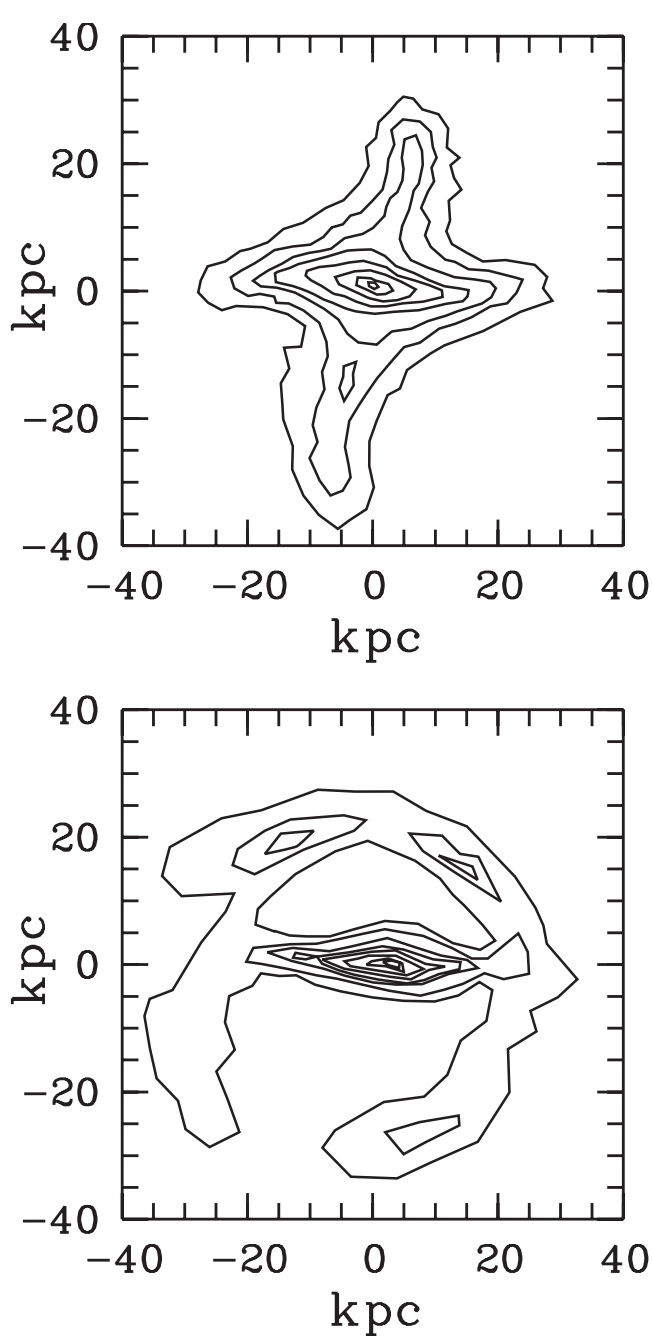

Fig. 10. Two projections of the stellar distribution in the simulated polar-ring galaxy (820 Myrs after the pericenter passage). The projection above (with the host galaxy seen nearly edge-on) has been chosen to fit the main characteristics of the observed system, as detailed in the text. The projection below, with the host disk seen edgeon and the polar ring face-on, is nearly orthogonal to the previous one. The contours are logarithmic, separated by a factor of 2 . The ring has rapidly become a closed structure, contrary to what happens in the minor merger scenario, and the mass distribution along the ring is still asymmetrical, which the major merger scenario fails to reproduce (see text).

the ring in AM 1934-563 is more extended than the northern one, the center of this "victim" galaxy should be south of the host galaxy, which should make the southern part of the polar ring brighter that the northern one. On the contrary, the northern part of the ring is brighter than the southern one. We have checked in simulations of $\mathrm{BC} 03$ that the more extended part of the ring is at least as luminous as the shortest part, when the ring is formed in a major merger.

We have explained above that the disruption of a small companion, during a minor merger, is unlikely to have formed the polar ring of AM 1934-563, for the ring would certainly appear unclosed, because the orbit of the companion is itself unclosed. Moreover, forming a massive enough ring, without disturbing the host disk too much, is a strong constraint on the mass of this companion.

On the contrary, the accretion scenario invoking a tidal mass transfer from a massive donor, without galaxy merger, has been shown to succeed in reproducing the characteristics of AM 1934-563. Furthermore, the small velocity dispersion of this group (see Sect. 3.1) could result from past galaxy interactions without merger, during which dynamical friction has dissipated the galaxy relative velocities. The velocity dispersion would be significantly higher if the three galaxies came from large distances and had not interacted yet. The small observed velocity dispersion also suggest that galaxies are close to their apocenter, confirming they have been closer, grazing in the past.

Which of the two other triplet members is the donor galaxy? Both galaxies are late-type and large enough to supply the matter to form the ring in AM 1934-563. The southern galaxy (PGC 399718) appears a more promising candidate, since it is perturbed and shows non-planar structures. Also, its red $B-V$ color can be a consequence of gas stripping during the interaction with AM 1934-563.

To confirm and to make more precise our model, new HI observations of the triplet are required, to search for a possible bridge between AM 1963-563 and the donor, and to estimate the HI content of the galaxies and of the ring. The extended southern part of the ring could be the origin of a bridge, as suggested by our numerical model (see the snapshot at $t=1150 \mathrm{Myr}$ in Fig. 9). Also 2D kinematical data for AM 1934-563 are needed to derive kinematical constraints on the formation scenario.

\section{Conclusions}

From our reported photometry and spectroscopic study of the AM 1934-563 system, we have studied a confirmed polar-ring galaxy (PRG). We have tested by numerical simulations the various possible scenarios, minor mergers, or tidal accretion of matter. It appears that the minor merger model does not reproduce the observed parameters in AM 1963-563. The tidal accretion of matter reproduces the overall morphology, including the inclination of the "polar" ring, its mass, and the S-shapes of the ring and the host disk. The small relative velocities of the three galaxies of the triplet may indicate that tidal interactions have occurred recently.

The case of accretion is quite likely for AM 1963-563, since it belongs to a triplet, with two possible donor galaxies. This is also the case for the prototype polar ring galaxy, NGC 4650A, which belongs to a dense group. The donor may also have left the group and be today further away from the PRG, because it can have a large relative velocity in this scenario. The small observed velocity dispersion in the group of AM 1963-563 may mean that we are seeing this flattened group face-on, so that the donor could have migrated away.

The present numerical simulations have shown that the matter exchanged between the donor and the target galaxy is not composed exclusively of gas, but a small number of stars are also accreted in the polar ring. This means that the age 
of the polar ring, estimated from the age of its stellar population, might be over-estimated, i.e. polar rings could be slightly younger than they appear. Yet, most of these accreted stars are found to be formed inside the tidal bridge that leads to ring formation, so the over-estimation of the age of a polar ring probably does not exceed $1-2 \times 10^{8} \mathrm{yr}$.

We also found that the host galaxy lies close to the Tully-Fisher relation. This is the case for most host galaxies of polar rings, and led Iodice et al. (2003) to the conclusion that PRGs contain dark matter along their polar structure. Unfortunately, the present observations do not allow us to study the ring kinematics in detail, thus we could not obtain constraints on its dark matter content. However, this PRG is a good target to study the distribution of dark matter, since the radius of the host galaxy and the polar ring are similar. Indeed, the too small radius of the host galaxy is an important problem in many PRGs. New HI or 2D-kinematical observations are required to study the distribution of dark matter.

In our simulations of the accretion scenario, we have assumed that gas is tidally accreted from a gas-rich donor. A variant of this scenario has been suggested by Cox et al. (2001): gas could form a ring via gradual accretion, and the stellar counterpart forms when a perturbation induces star formation. At this stage, we do not discriminate between these two possibilities: we only want to know whether the ring is formed by gas accretion in general, or by galaxy interaction/mergers.

The case of AM 1934-563 (like other PRGs according to $\mathrm{BC03}$ ) appear to support the view that galaxies are not formed in one event only, but they acquire mass all along their lives, as is also attested by morphological properties of disk galaxies, like bars (Bournaud \& Combes 2002) and lopsidedness (Bournaud et al. 2005b). Material accreted during the life of galaxies is quite visible in the case of PRGs because of its peculiar perpendicular orientation. In the most general case, the material will be accreted in the plane, or with some angle lower than about $60^{\circ}$ from the plane, which can give rise to warps, disk lopsidedness of the disk or density waves like arms or bars.

Acknowledgements. This work was done while VR was visiting LERMA in Paris, thanks to a CNRS 3-months visitor grant. V.R. acknowledges support from the Russian Foundation for Basic Research (03-02-17152) and from the Russian Federal Program "Astronomy" (40.022.1.1.1101). M.F.-A. and M. de O.-A. acknowledge the partial support of the Fundação de Amparo ã Pesquisa do Estado de Minas Gerais (FAPEMIG) and the Ministério da Ciência e Tecnologia (MCT, Brazil). We would like to thank the anonymous referee whose detailed remarks have helped improve the paper. The computations in this work were carried out on the Fujitsu NEC-SX5 of the CNRS computing center, at IDRIS. This research has made use of the NASA/IPAC Extragalactic Database (NED) which is operated by the Jet Propulsion Laboratory, California Institute of Technology, under contract with the National Aeronautics and Space Administration. We made use of the LEDA database (http://leda.univ-lyon1.fr).

\section{References}

Afanasiev, V. L., Dodonov, S. N., \& Moiseev, A. V. 2001, in Stellar Dynamics: From Classic to Modern, ed. L. P. Ossipkov, \& I. I. Nikiforov, St. Petersburg, 103

Armus, L., Heckman, T. M., \& Miley, G. K. 1990, ApJ, 364, 471

Bekki, K. 1998, ApJ, 499, 635

Bicker, J., Fritze-v.Alvensleben, U., Moller, C. S., \& Fricke, K. J. 2004, A\&A, 413, 37

Brocca, C., Bettoni, D., \& Galletta, G. 1997, A\&A, 326, 907

Bournaud, F., \& Combes, F. 2003, A\&A, 401, 817 (BC03)

Bournaud, F., Combes, F., \& Jog, C. J. 2004, A\&A, 418, L27

Bournaud, F., Jog, C. J., \& Combes, F. 2005a, A\&A, 437, 69

Bournaud, F., Combes, F., Jog, C. J., \& Puerari, I. 2005b, A\&A, 438, 507

Bushouse, H. A. 1987, ApJ, 320, 49

Buta, R., Mitra, S., de Vaucouleurs, G., \& Corwin, H. G. 1994, AJ, 107, 118

Buta, R., \& Williams, K. L. 1995, AJ, 109, 543

Cox, A. L., Sparke, L. S., Watson, A. M., \& van Moorsel, G. 2001, AJ, 121, 692

de Jong, R. S. 1996, A\&A, 313, 377

de Grijs, R. 1998, MNRAS, 299, 595

Fairall, A. P. 1992, Observatory, 112, 286

Graham, J. A. 1982, PASP, 94, 244

Ho, L. C., Filippenko, A. V., \& Sargent, W. L. W. 1995, ApJS, 98, 477

Iodice, E., Arnaboldi, M., Sparke, L. S., \& Freeman, K. C. 2002, A\&A, 391, 117

Iodice, E., Arnaboldi, M., Bournaud, F., et al. 2003, ApJ, 585, 730

Iodice, E., Arnaboldi, M., Sparke, L. S., et al. 2004, A\&A, 418, 41

James, R. A. 1977, J. Comput. Phys., 25, 71

Kannappan, Sh. J., Fabricant, D. G., \& Franx, M. 2002, AJ, 123, 2358

Karachentseva, V. E., \& Karachentsev, I. D. 2000, Astron. Rep., 44, 501

Landolt, A. U. 1983, AJ, 88, 439

Matthews, L. D., \& de Grijs, R. 2004, AJ, 128, 137

Moiseev, A. V. 2002, Astron. Lett., 28, 755

Monnet, G., \& Simien, F. 1977, A\&A, 56, 173

Reshetnikov, V. P. 2004, A\&A, 416, 889

Reshetnikov, V. P., Hagen-Thorn, V. A., \& Yakovleva, V. A. 1994, A\&A, 290, 693

Reshetnikov, V. P., Faúndez-Abans, M., \& de Oliveira-Abans, M. 2001, MNRAS, 322, 689

Reshetnikov, V., \& Sotnikova, N. 1997, A\&A, 325, 933

Schlegel, D. J., Finkbeiner, D. P., \& Davis, M. 1998, ApJ, 500, 525

Schweizer, F., Whitmore, B. C., \& Rubin, V. C. 1983, AJ, 88, 909

Shalyapina, L. V., Moiseev, A. V., Yakovleva, V. A., et al. 2004, Astron. Lett., 30, 1

Skrutskie, M. F., Schneider, S. E., Steining, R., et al. 1997, in The Impact of Large Scale Near-IR Sky Surveys, ed. F. Garzon et al. (Dordrecht: Kluwer), 25

Tully, R. B., Pierce, M. J., Huang, J.-Sh., et al. 1998, AJ, 115, 2264

van Driel, W., Combes, F., Arnaboldi, M., \& Sparke, L. S. 2002, A\&A, 386,140

Veilleux, S., \& Osterbrock, D. E. 1987, ApJS, 63, 295

Whitmore, B. C., Lucas, R. A., McElroy, D. B., et al. 1990, AJ, 100, 1489 (PRC) 